\title{
Pulsed Electromagnetic Fields to Influence Pain and Muscle Healing Following Muscle Injury in Rats
}

\author{
Hyun-Mo Koo ${ }^{1}$, Sang-Su Na ${ }^{2}$, and Min-Sik Yong ${ }^{3 *}$ \\ ${ }^{1}$ Department of Physical Therapy, College of Science, Kyungsung University, Busan 48434, Korea \\ ${ }^{2}$ Department of Physical Therapy, Daegu University, Gyungbuk 38453, Korea \\ ${ }^{3}$ Department of Physical Therapy, Youngsan University, Gyungnam 50510, Korea
}

(Received 21 July 2015, Received in final form 10 August 2015, Accepted 10 August 2015)

\begin{abstract}
Muscle contusion has a negative effect on muscle function. Although several studies showed that pain control and muscle recovery is facilitated by pulsed electromagnetic fields (PEMF), there has not been much research regarding the specific effects of PEMF on them. The aim of the present study is to investigate effects of PEMF on pain and muscle recovery following extensor digitorum longus (EDL) contusion injury through measuring the expression of the c-fos proto-oncogene and nerve growth factor (NGF). Significantly reduced c-fos expression in the spinal cord was shown in PEMF groups compared with control (CON) groups. There was no significant difference between PEMF1 and CON1, but significantly increased NGF expression was shown in PEMF3 and PEMF5 compared with in CON groups, where the numbers in the group names are the days from contusion. In conclusion, PEMF could be used to not only reduce pain in muscle injuries by down-regulating cfos expression in the spinal cord, but it could also influence muscle healing through increasing NGF expression in the injured muscle.
\end{abstract}

Keywords : pulsed electromagnetic fields, neurotrophic factor, muscle injury, pain

\section{Introduction}

Muscle can be impaired by various conditions such as strain, contusions, crushing, or lacerations. Among many types of muscular impairments, it is known that muscle contusion accounts for more than $90 \%$ of all injuries associated with sports. Since a muscle contusion results from a heavy and a sudden compressive force to the muscle, it tends to occur frequently in contact sports $[1$, 2]. In addition, this kind of muscle trauma has a negative effect on muscle function by leading to problems such as atrophy, contracture, and pain [3].

Controlling pain in the early stage is necessary to restore function following muscle injuries and it affects the degree of rehabilitation in the later stage [4]. C-fos, one of the immediate-early genes expressed from noxious stimuli, has been regarded as a marker of nociceptive processes. In many studies, the expression of c-fos protein has been examined to evaluate nociceptive response in the

CThe Korean Magnetics Society. All rights reserved.

*Corresponding author: Tel: +82-55-380-9367

Fax:+82-55-380-9305, e-mail: yongms@ysu.ac.kr spinal cord $[1,5,6]$.

The healing of an injured muscle occurs through several processes including formation of connective tissue scar, regeneration of myofibers and intramuscular nerves, and vascularization. These processes related to muscle healing could be influenced by the secretion of various growth factors [7]. Neurotrophins, a family of growth factors including brain-derived neurotrophic factor (BDNF), neurotrophin-3 (NT-3), and nerve growth factor (NGF), are known to stimulate neuronal growth, survival, and differentiation, and those functions are mediated by binding to neurotrophin receptors. Neurotrophins and their receptors are mainly expressed not only in nervous tissues, but also in non-nervous tissues such as muscle or vasculature [8]. In particular, it is suggested that NGF is capable of maintaining skeletal muscle fibers and could elicit differentiation and an anti-apoptotic effect [9].

Pulsed electromagnetic fields (PEMF), characterized by extremely low frequencies between 5 and $300 \mathrm{~Hz}$, are known as one of the physical agents used in the field of physical therapy, and many studies have reported its therapeutic effects with various pathologic conditions such as fracture, spinal cord injury, and musculoskeletal 
disorders [4, 10-12]. Although several studies showed that pain control and muscle recovery is facilitated by applying PEMF, there has not yet been much research regarding the specific effects of PEMF on them.

In this regard, the aim of the present study is to investigate whether the application of PEMF affects pain and muscle recovery following extensor digitorum longus (EDL) contusion injury.

\section{Materials and Methods}

\subsection{Animals}

30 male Sprague-Dawley rats ( 8 weeks old, with body weight 250-300 g) were used and maintained at a 12 hour on/12 hour off light/dark cycle with ad libitum access to food and water. All the experiments were performed in accordance with protocols approved by the Animal Experiment Committee in Daegu University, based on the NIH Guidelines for the Care and Use of Laboratory Animals (NIH publication, 1996). All rats were randomly divided into 6 groups: CON1 (including rats sacrificed 1 day after surgery, $n=5$ ), $C O N 3$ (including rats sacrificed 3 days after surgery, $\mathrm{n}=5$ ), CON5 (including rats sacrificed 5 days after surgery, $\mathrm{n}=5$ ), PEMF1 (including rats with applied PEMF for 1 day after surgery, $n=5$ ), PEMF3 (including rats with applied PEMF for 3 days after surgery, $\mathrm{n}=5$ ), and PEMF5 (including rats with applied PEMF for 5 days after surgery, $n=5$ ).

\subsection{Experimental procedure}

Muscle contusion was induced on the right EDL muscle in all animals. In order to apply PEMF to the injured site, a Diapulse machine (Diapulse Corp., USA) was used at a frequency of $27.12 \mathrm{MHz}$ with 5 gauss intensity for 20 minutes per day.

\subsection{Sampling and western blot analysis}

The right EDL muscles and the spinal cords of each group were collected, washed twice in PBS, and then homogenized and lysed with a buffer $(137 \mathrm{mM} \mathrm{NaCl}, 8.1$ $\mathrm{mM} \mathrm{Na} \mathrm{HPO}_{4}, 2.7 \mathrm{mM} \mathrm{KCl}, 1.5 \mathrm{mM} \mathrm{KH} \mathrm{PO}_{4}, 2.5 \mathrm{mM}$ EDTA, $1 \mathrm{mM}$ dithiothreitol, $0.1 \mathrm{mM}$ PMSF, $10 \mu \mathrm{g} / \mathrm{ml}$ leupeptin [pH 7.5]) for $30 \mathrm{~min}$ on ice. The lysates were centrifuged for $10 \mathrm{~min}$ at $15,000 \mathrm{rpm}$ at $4^{\circ} \mathrm{C}$, and the protein concentration was determined. Equal amounts of protein $(40 \mu \mathrm{g})$ were resolved via $10 \%$ sodium dodecyl sulfate-polyacrylamide gel electrophoresis (SDS-PAGE) and transferred to nitrocellulose membranes. The blots were washed with TBST $(10 \mathrm{mM}$ Tris· $\mathrm{HCl}[\mathrm{pH}$ 7.6], 150 $\mathrm{mM} \mathrm{NaCl}, 0.05 \%$ Tween 20 ), blocked with $5 \%$ skim milk for 1 hour, and then incubated with the appropriate primary antibodies at the dilutions recommended by the suppliers. The membranes were washed, and the primary antibodies were detected using horseradish peroxidaseconjugated goat anti-rabbit IgG or goat-anti mouse IgG. The bands were then visualized via enhanced chemiluminescence (Amersham Pharmacia Biotech, Piscataway, NJ, USA).

\subsection{Statistical analysis}

The results were expressed as the means \pm standard error (SE). All experiments were analyzed via analysis of variance, and Fisher's LSD test was used for post hoc evaluations. The difference is considered statistically significant when $p<0.05$.

\section{Results and Discussion}

Although PEMF has been used as a therapeutic method for various medical conditions over the last 40 years, effects of PEMF might have a controversial aspect because researches using PEMF show inconsistent conclusions $[10,13]$. Mollan et al. concluded that the application of PEMF did not influence long-bone fracture healing [14]. Varken et al. reported that applying PEMF to knee osteoarthritis did not improve pain control or joint stiffness [15].

However, there are also other researches demonstrating beneficial effects of PEMF. Ganesan et al. reported that PEMF was involved in the recovery from arthritis [16]. Kang et al. concluded that PEMF was an effective method for preventing amyotrophy following spinal cord injury [11]. In order to discern an effect of PEMF more clearly, the present study investigated whether PEMF can improve pain control and muscle recovery through the use of a rat model with muscle contusion.

From the results, it was found that significantly reduced c-fos expression in the spinal cord was shown in PEMF groups compared with CON groups (Fig. 1). The expression of c-fos in the spinal cord can be used as an indirect marker that is involved in nociceptive signaling. In various pain models, it is demonstrated that administration of several substances such as MK-801, ketamine, and memantine can influence the degree of nociceptive behavior through suppression of spinal c-fos expression. In contrast, c-fos expression can be up-regulated by several factors that induce pain such as stress or inflammation $[5,6]$. Judging from these facts, the results of the present study imply that the application of PEMF may induce an analgesic effect on injured muscle in the acute phase.

In the present study, NGF expression induced by apply- 

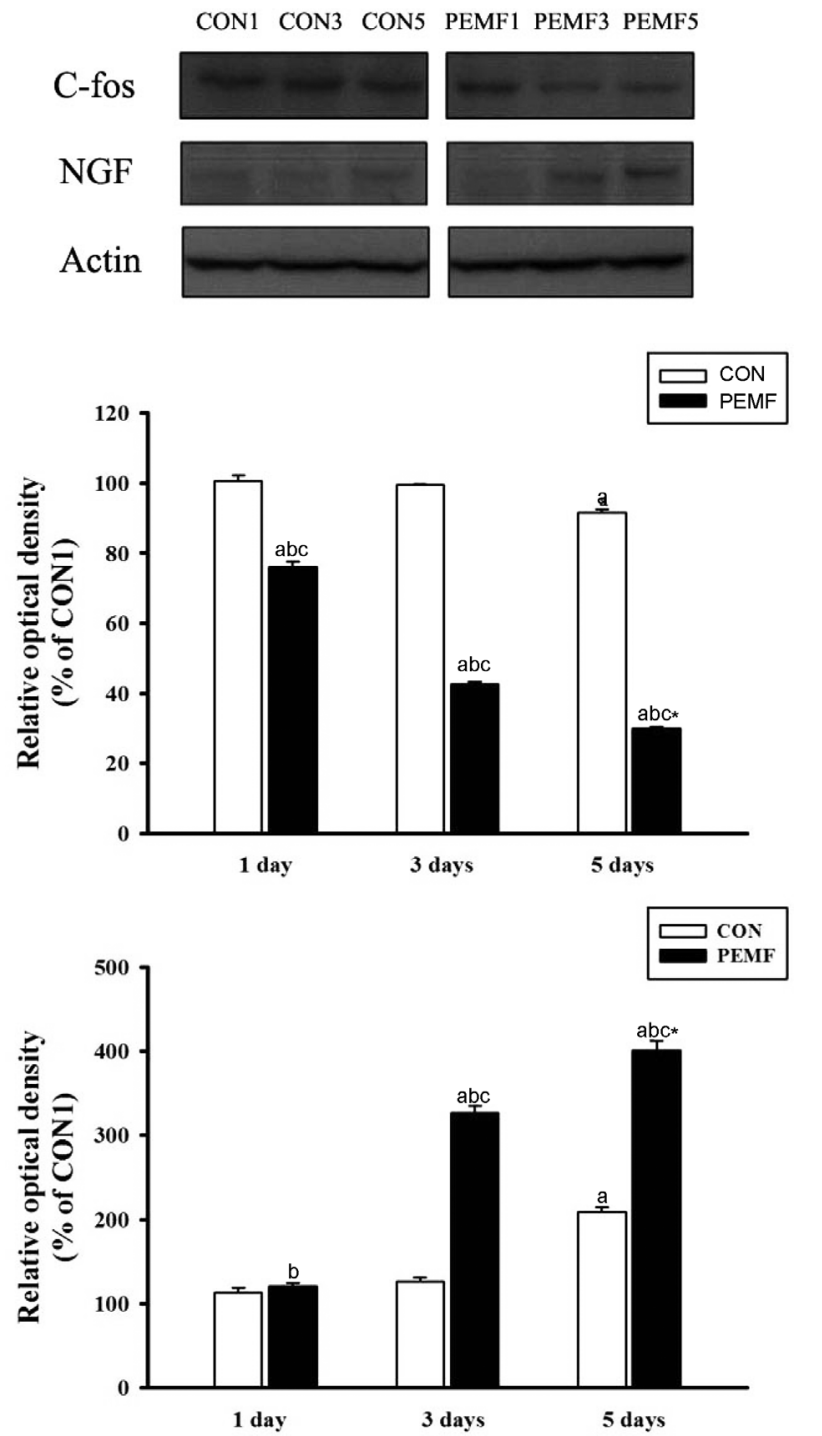

Fig. 1. Western blot analysis for c-fos expression in rat spinal cord and NGF expression in rat EDL muscle in each group. The values represent the means \pm SE. ap $<.05$ versus $\mathrm{CON} 3$, $\mathrm{bp}<.05$ versus CON5, $\mathrm{cp}<.05$ versus PEMF1, ${ }^{*} \mathrm{p}<.05$ versus PEMF3.

ing PEMF was also examined. There was no significant difference between PEMF1 and CON1, but significantly increased NGF expression was shown in PEMF3 and PEMF5 compared with the corresponding CON groups (Fig. 1). To date, it has been generally known that neurotrophins play a role in regulating several factors including neuronal survival, plasticity, growth, and death. However, accumulating evidence suggests that neurotrophins influence the development, function, and regeneration of skeletal muscle [17]. It is suggested that NGF is involved in muscle regeneration among various neurotrophins in particular. Lavasani et al. reported that NGF improves the muscle regeneration capacity of muscle stem cells. Furthermore, it might be possible that NGF triggers and otherwise facilitates the muscle regeneration process [18].

The muscle healing process occurring after injury consists of several different phases, including degeneration and inflammation, regeneration, and the development of fibrosis. Muscle healing can be facilitated not only by enhancing muscle regeneration, but also by preventing muscle fibrosis [7]. The results of the present study suggest that PEMF-induced NGF expression might affect the muscle regeneration phase, thereby leading to an effective muscle healing.

Consequently, the present study suggests that PEMF could be used to reduce pain in muscle injuries by downregulating c-fos expression in the spinal cord. It is also suggested that PEMF could have positive influences on muscle healing through increasing NGF expression in the injured muscle.

\section{References}

[1] M. H. Kim and S. H. Cheon, J. Magn. 17, 68 (2012).

[2] T. A. Jarvinen, T. L. Jarvinen, M. Kaariainen, H. Kalimo, and M. Jarvinen, Am. J. Sports Med. 33, 745 (2005).

[3] C. D. Markert, M. A. Merrick, T. E. Kirby, and S. T. Devor, Arch. Phys. Med. Rehabil. 86, 1304 (2005).

[4] G. L. Cheing, J. W. Wan, and S. Kai Lo, J. Rehabil Med. 37, 372 (2005).

[5] L. Quintero, M. C. Cuesta, J. A. Silva, J. L. Arcaya, L. Pinerua-Suhaibar, W. Maixner, and H. Suarez-Roca, Brain Res. 965, 259 (2003).

[6] G. H. Zhang, S. S. Min, K. S. Lee, S. K. Back, S. J. Yoon, Y. W. Yoon, Y. I. Kim, H. S. Na, S. K. Hong, and H. C. Han, Anesth Analg. 99, 152 (2004).

[7] J. Huard, Y. Li, and F. H. Fu, J. Bone Joint Surg. Am. 84, 822 (2002).

[8] H. Kim, Q. Li, B. L. Hempstead, and J. A. Madri, J. Biol. Chem. 279, 33538 (2004).

[9] G. Chevrel, R. Hohlfeld, and M. Sendtner, Muscle Nerve. 33, 462 (2006).

[10] K. Hug and M. Roosli, Bioelectromagnetics 33, 95 (2012).

[11] J.-H. Kang, S.-Y. Park, and Y.-S. Lee, J. Magn. 16, 253 (2011).

[12] D. Galace de Freitas, F. B. Marcondes, R. L. Monteiro, S. G. Rosa, P. Maria de Moraes Barros Fucs, and T. Y. Fukuda, Arch. Phys. Med. Rehabil. 95, 345 (2014).

[13] N. M. Shupak, F. S. Prato, and A. W. Thomas, Radio Science Bulletin 307, 9 (2003).

[14] B. Mollon, V. da Silva, J. W. Busse, T. A. Einhorn, and M. Bhandari, J. Bone Joint Surg. Am. 90, 2322 (2008).

[15] P. Vavken, F. Arrich, O. Schuhfried, and R. Dorotka, J. Rehabil Med. 41, 406 (2009). 
[16] K. Ganesan, A. C. Gengadharan, C. Balachandran, B. M. Manohar, and R. Puvanakrishnan, Indian J. Exp. Biol. 47, 939 (2009).

[17] C. Clow and B. J. Jasmin, Mol. Biol. Cell 21, 2182
(2010).

[18] K. Sakuma and A. Yamaguchi, J. Biomed Biotechnol. 2011, 201696 (2011). 